\title{
Influence of the (n-n')-mixing processes on the optical properties of the hydrogen clouds in the broad-line region of AGNs
}

\author{
M.S. Dimitrijević ${ }^{1,2}$, V.A. Srećković ${ }^{3}$ and Lj.M. Ignjatović ${ }^{3}$ \\ 1 Astronomical Observatory, Volgina 7, 11060 Belgrade, Serbia \\ 2 Sorbonne Université, Observatoire de Paris, Université PSL, CNRS, \\ LERMA, F-92190 Meudon, France (E-mail: mdimitrijevic@aobrs) \\ 3 Institute of Physics Belgrade, Pregrevica 118, 11080 Zemun, Belgrade, \\ Serbia,(E-mail:vlada@ipb.ac.rs)
}

Received: July 16, 2019; Accepted: October 29, 2019

\begin{abstract}
The role of $\left(n-n^{\prime}\right)$-mixing processes in active galactic nuclei broadline region (BLR) clouds has been studied. Our investigation indicates that these collisional processes must have influence on the populations of the excited hydrogen atoms in moderately ionized layers of dense parts of the BLR clouds and are of interest for its modelling and research. We provide results potentially important for the spectroscopy and modeling of the BLR clouds, as well as for the study of hydrogen Rydberg states.
\end{abstract}

Key words: Galaxies: active - atomic processes -Plasmas

\section{Introduction}

In the papers Mihajlov et al. (2005); Gnedin et al. (2009); Mihajlov et al. (2016) authors drew attention to the importance of inelastic processes in slow collisions of Rydberg state atoms with ground state hydrogen atoms (i.e. excitation/deexcitation processes) in solar and stellar atmospheres

$$
\mathrm{H}^{*}(n)+\mathrm{H} \rightarrow \mathrm{H}+\mathrm{H}^{*}\left(n^{\prime}\right),
$$

where $\mathrm{H}$ are hydrogen atoms in their ground states, $\mathrm{H}^{*}(n)$ and $\mathrm{H}^{*}\left(n^{\prime}\right)$ are hydrogen atoms in a highly excited (Rydberg) state.

These authors concluded that $\left(n-n^{\prime}\right)$-mixing processes (Eq. 1) significantly influence on the populations of hydrogen Rydberg atoms (RA) in weakly ionized layers of stellar atmospheres and particularly in Solar photosphere and the lower chromosphere and need to be added in any modelling and investigation of these regions. In Barklem (2007) and Mashonkina (2009) the $\left(n-n^{\prime}\right)$-mixing processes have also been analyzed together with others, and the conclusion was that there is a great need for their further investigation, since in many cases there are uncertainties in the rate coefficients of hydrogen collisions (see also Srećković et al., 2018a,b). 
In active galactic nuclei (AGN), especially in the region of the moderately ionized layers of dense parts of the broad-line region (BLR), in which physical conditions are closer to stellar atmospheres than to photoionized nebulae (Ilić et al., 2007; Negrete et al., 2012; Ilić et al., 2017; Bon et al., 2018; Osterbrock \& Ferland, 2006). In the BLR clouds temperature may drop to the much lower values where the gas is weakly ionized with huge number of hydrogen atoms in excited states, due to the high density and large optical depths (Crosas \& Weisheit, 1993; Negrete et al., 2012; Netzer, 2013). Consequently, it is of interest to investigate the influence of the mentioned collisional processes Srećković et al. (2018c).

That is the reason why we have extended the investigation of these processes and calculated their rate coefficients for a wider region of plasma parameters and for quantum numbers $\mathrm{n}$ up to 20 in order to be potentially used in AGN modeling. The results presented here cover the range of temperatures $2000 \mathrm{~K} \leq T \leq 30000 \mathrm{~K}$, broader than the regions relevant for weakly ionized layers of dense parts of the BLR clouds (see e.g. Crosas \& Weisheit, 1993; Negrete et al., 2012), so that the data presented here can be also used for investigation of the atmospheres of Sun and Sun-like stars etc. (Mihajlov et al., 2011; Mihajlov et al., 2011; Srećković et al., 2014). Also, study of these processes are of interest for some laboratory spectroscopical investigation and technical applications (Dubernet et al., 2016; Marinković et al., 2017).

We describe in Section 2 the methods of calculation and then in Section 3 we give the results of the calculation. In Section 4 the conclusions are presented.

\section{Method}

\subsection{The resonant mechanism}

On the basis of the resonant mechanism (Mihajlov et al., 2012; Srećković et al., $2013,2018 \mathrm{c}$ ) the $\left(n-n^{\prime}\right)$-mixing processes (Eq. 1$)$ are characterized as a product of the resonant energy exchange between the outer electron $e$ and the electronic component of the subsystem $\mathrm{H}^{+}+\mathrm{H}$ (Fig.1). The system $\mathrm{H}^{*}(n)+\mathrm{H}$ is described as: $e+\left(\mathrm{H}^{+}+\mathrm{H}\right)$, where $e$ is the outer electron of the hydrogen $\mathrm{RA} \mathrm{H}^{*}(n)$. For describing the subsystem $\left(\mathrm{H}^{+}+\mathrm{H}\right)$ electronic states, the adiabatic electronic ground state, or the first excited state of the molecular ion were used.

\subsection{The (n-n')-mixing rate coefficients}

The procedure of obtaining the rate coefficients for the processes (Eq. 1) is described in detail in Mihajlov et al. (2004); Srećković et al. (2013) and only the corresponding final expressions are given here. The rate coefficients $K_{n ; n+p}^{\operatorname{mix}}(T)$ for the mixing processes between two excited levels with main quantum numbers 


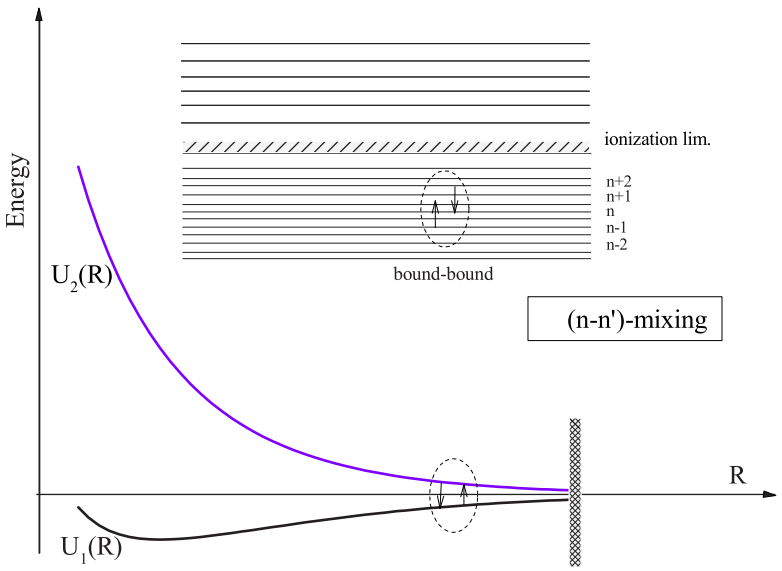

Figure 1. The schematic presentation of the (n-n')-mixing processes. $R$ presents the internuclear distance, $U_{1}(R)$ and $U_{2}(R)$ are the potential energy curves of the initial and final electronic state of considered system.

$n$ and $n+p$, can be expressed as a function of the temperature $T$ as

$$
\begin{aligned}
& K_{n ; n+p}^{\operatorname{mix}}(T)=\frac{2 \pi}{3 \sqrt{3}} \frac{\left(e a_{0}\right)^{2}}{\hbar} \cdot n^{-5} \cdot g_{n ; n+p} \times \\
& \times \int_{R_{\min }(n, n+p)}^{R_{\max }(n, n+p)} X(R) \cdot \exp \left[-\frac{U_{2}(R)}{k T}\right] \frac{R^{4} \cdot d R}{a_{0}^{5}},
\end{aligned}
$$

where $T_{e}=T_{a}=T, T_{e}$ and $T_{a}$ are the electron and atom temperatures and $T$ is their common value, $g_{n ; n+p}$ is the Gaunt factor, $R_{\min }(n, n+p)$ and $R_{\max }(n, n+$ $p$ ) are quantities defined in Mihajlov et al. (2008), $a_{0}$ is the atomic unit of length, $e$ is the value of electron charge, $R$ is the internuclear distance, $\hbar$ and $k$ are the Planck and Boltzmann constants and $U_{2}(R)$ is the potential curve of the first excited electronic states of the considered system. $X(R)$ is defined as the function $X(R)=\frac{\Gamma\left(3 / 2 ; \frac{\left|U_{1}(R)\right|}{k T}\right)}{\Gamma(3 / 2)}$, where $\Gamma(3 / 2 ; x)$ and $\Gamma(3 / 2)$ are the corresponding values of the incomplete and complete Gamma functions. The rate coefficients for the corresponding inverse deexcitation processes are

$$
K_{n ; n-p}^{m i x}(T)=K_{n-p ; n}^{m i x}(T) \cdot \frac{(n-p)^{2}}{n^{2}} \cdot \exp \left(\frac{\epsilon_{n-p ; n}}{k T}\right),
$$

where $\epsilon_{n-p ; n}=\epsilon_{n}-\epsilon_{n-p}$ and $\epsilon_{n}=-R y / n^{2}, R y$ is the Rydberg constant (for details see Mihajlov et al., 2008). 


\subsection{The relative efficiency}

To estimate the relative efficiency of processes defined in Eq. (1) in comparison to concurrent electron-atom mixing processes i.e. electron-excited atom collisional excitation/de-excitation processes

$$
e+\mathrm{H}^{*}(n) \rightarrow e^{\prime}+\mathrm{H}^{*}\left(n^{\prime}=n \pm p\right),
$$

we use the parameter $F_{n}^{( \pm)}(T)$

$$
F_{n}^{( \pm)}(T)=\frac{\sum_{p=1}^{5} K_{n ; n \pm p}^{m i x}(T) N(n) N(1)}{\sum_{p=1}^{5} K_{n ; n \pm p}^{e a}(T) N(n) N_{e}}=\frac{\sum_{p=1}^{5} K_{n ; n \pm p}^{m i x}(T)}{\sum_{p=1}^{5} K_{n ; n \pm p}^{e a}(T)} \eta
$$

where $N(n)$ is the population of the excited atom states, $N_{e}$ is the density of free electrons and the parameter $\eta=N(1) / N_{e}$. Here quantity $K_{n ; n \pm p}^{e a}\left(T_{e}=T\right)$ is the rate coefficient for the electron-atom process (Eq.4) taken from Vriens \& Smeets (1980). The expressions $K_{n ; n \pm p}^{m i x}(T) N(n) N(1)$ and $K_{n ; n \pm p}^{e a}(T) N(n) N_{e}$ are the partial atom- and electron-Rydberg atom excitation and deexcitation fluxes.

\section{Results and Discussion}

The rate coefficients $K_{n ; n \pm p}^{\operatorname{mix}}(T)$ for mixing processes (Eq.1) are calculated for the following parameters $4 \leq n \leq 20,1 \leq p \leq 5$ and $2000 \mathrm{~K} \leq T \leq 30000 \mathrm{~K}$. Here we give the results only for temperatures $\leq 10000 \mathrm{~K}$, which are relevant for the moderately ionized layers of dense parts of the BLR clouds and data for higher temperatures are of interest for other moderately ionized astrophysical plasmas. In Fig. 2 the excitation rate coefficients are presented for selected excited states $n=4, \ldots 13$ and temperature $T$ in the range $3000-10000 \mathrm{~K}$ relevant for the moderately ionized layers of dense parts of the BLR clouds.

We have calculated the values of the parameter $F_{n}^{(+)}$for $4 \leq n \leq 8$ in order to compare the relative influence of $\left(n-n^{\prime}\right)$-mixing processes (Eq.1) and influence of concurrent electron-atom excitation processes (Eq.4) for the physical conditions which could be relevant in the BLR of AGNs (see Fig. 3). It can be noted that the $\left(n-n^{\prime}\right)$-mixing processes for $n \leq 5$ are dominating over the concurrent ones in the lower temperature regime (Eq.4, bottom panel) whereas for $6 \leq n \leq 8$ both processes are comparable (see Fig. 3). Also the processes (Eq.1) are comparable with concurrent excitation processes (Eq.4) only for lower $n$ in the parts of the BLR with higher temperatures. On Fig. 3 the shaded area $(2 \leq n \leq 4)$ present extrapolated values due to the missing reliable data for the corresponding rate coefficients. The only existing rate coefficients in the literature for $n=2$ and 3 are data from Drawin (1968) which are not recommended (see Barklem 2007, Srećković et al. 2018c). We note that these rate coefficients for $n=2$ and 3 are of great importance and the impact of processes (see Eq.1) should be much 


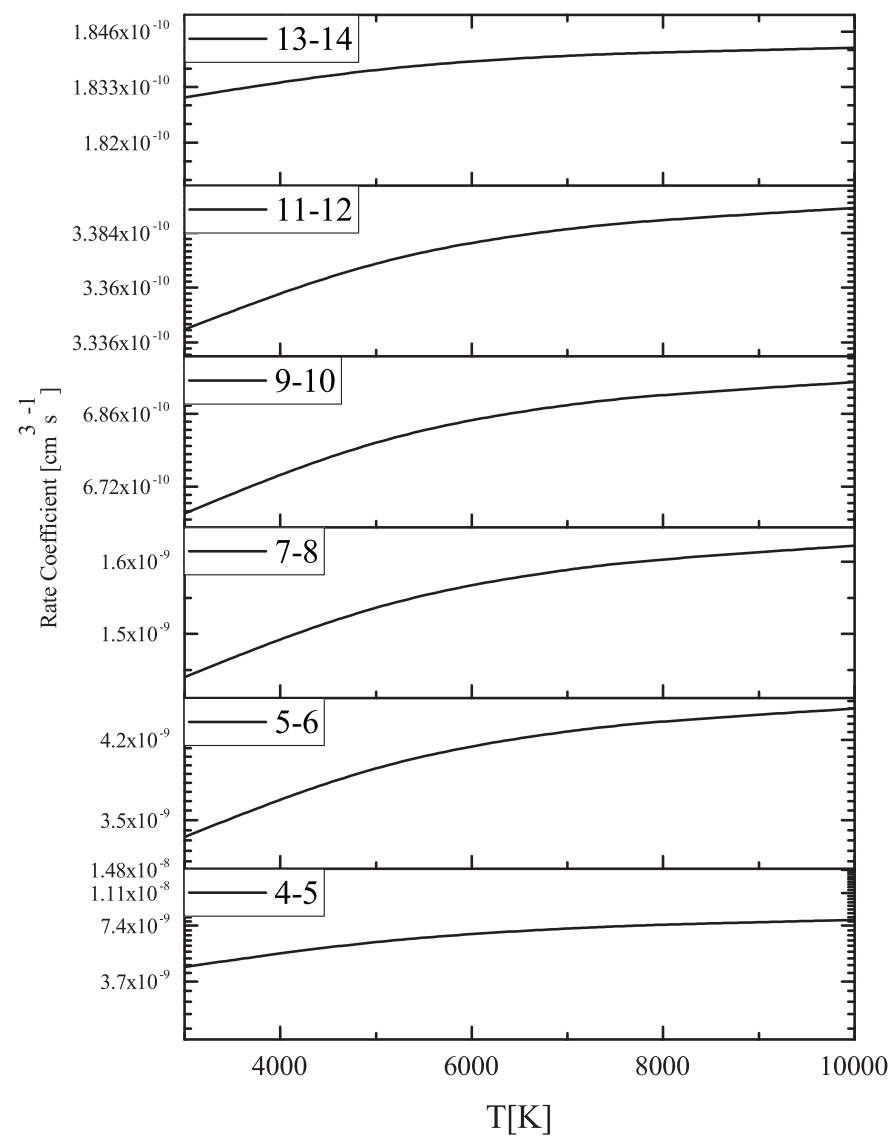

Figure 2. Plot of excitation rate coefficients for processes $\mathrm{H}^{*}(n)+\mathrm{H} \rightarrow \mathrm{H}+\mathrm{H}^{*}\left(n^{\prime}\right)$, for selected excited states $n=4, \ldots 13$ and temperature $T$ in the range $3000-10000 \mathrm{~K}$.

higher on the populations of hydrogen excited atoms of the AGNs in comparison to the concurrent processes.

The presented results demonstrate that these processes must have a noticeable influence on the optical properties of the weakly ionized regions of dense parts of the BLR clouds and that they should be used in the models. In addition, they could be helpful for modeling of various stellar atmospheres with low effective temperatures, as well as for the research of Rydberg states of hydrogen and the analysis of their influence during the cosmological recombination epoch 


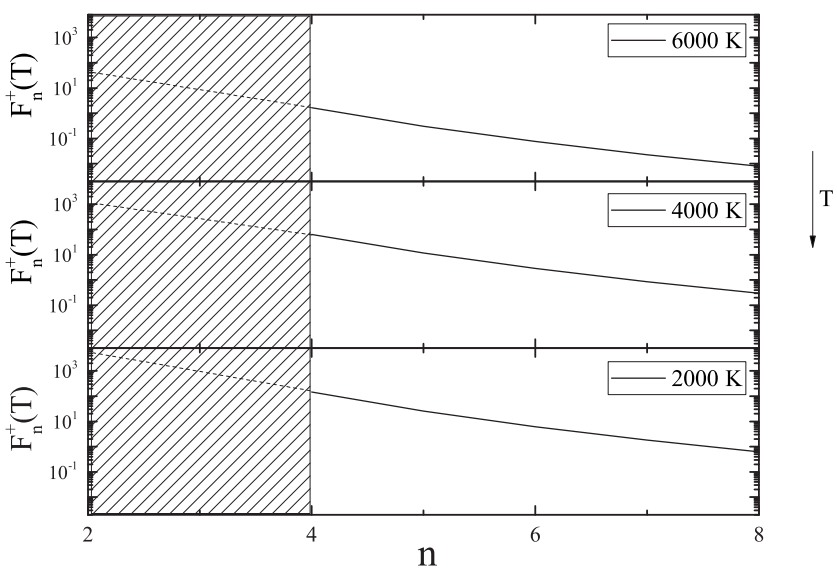

Figure 3. Efficiencies $F_{n}^{(+)}$of the analyzed processes for $4 \leq n \leq 8$ and temperatures $6000 \mathrm{~K}$ (upper panel), $4000 \mathrm{~K}$ (middle panel), and $2000 \mathrm{~K}$ (bottom panel). The extrapolated values below $n=4$ (see text) are shown in the left shaded region.

(Fontenla et al., 2009; Przybilla \& Butler, 2004; Barklem, 2007; Mashonkina, 2009).

\section{Conclusion}

Our primary task in this paper was determination of rate coefficients for parameters that exists in BLR clouds in order to be used in the corresponding models. The results demonstrate the fact that the considered (n-n')-mixing processes must have a significant influence on the populations of excited hydrogen atoms in cooler and denser parts of the BLR clouds in AGNs in comparison to the concurrent processes and must have influence on the optical properties.

Acknowledgements. This work is made within projects of the Ministry of Education, Science and Technological Development of Serbia, 176002, III44002.

\section{References}

Barklem, P. S., Non-LTE Balmer line formation in late-type spectra: effects of atomic processes involving hydrogen atoms. 2007, Astron. Astrophys., 466, 327

Bon, N., Bon, E., \& Marziani, P., AGN Broad Line Region variability in the context of Eigenvector 1: case of NGC 5548. 2018, Frontiers in Astronomy and Space Sciences, $\mathbf{5}, 3$ 
Crosas, M. \& Weisheit, J. C., Hydrogen molecules in quasar broad-line regions. 1993, Mon. Not. R. Astron. Soc., 262, 359

Drawin, H.-W., Zur formelmäßigen Darstellung des Ionisierungsquerschnitts für den Atom-Atomstoß und über die Ionen-Elektronen-Rekombination im dichten Neutralgas. 1968, Z. Phys., 211, 404

Dubernet, M.-L., Antony, B., Ba, Y.-A., et al., The virtual atomic and molecular data centre (VAMDC) consortium. 2016, J. Phys. B, 49, 074003

Fontenla, J. M., Curdt, W., Haberreiter, M., Harder, J., \& Tian, H., Semiempirical Models of the Solar Atmosphere. III. Set of Non-LTE Models for FarUltraviolet/Extreme-Ultraviolet Irradiance Computation. 2009, Astrophys. J., 707, 482

Gnedin, Y. N., Mihajlov, A., Ignjatović, L. M., et al., Rydberg atoms in astrophysics. 2009, New Astron. Rev., 53, 259

Ilić, D., Popović, L. Č., Ciroi, S., La Mura, G., \& Rafanelli, P., Physical Properties of the BLR of AGN: Boltzmann-Plot vs. CLOUDY Models. 2007, in American Institute of Physics Conference Series, Vol. 938, Spectral Line Shapes in Astrophysics, ed. L. C. Popovic \& M. S. Dimitrijevic, 70-75

Ilić, D., Shapovalova, A. I., Popović, L. Č., et al., Long-term monitoring of the broadline region properties in a selected sample of AGN. 2017, Frontiers in Astronomy and Space Sciences, 4, 12

Marinković, B. P., Jevremović, D., Srećković, V. A., et al., BEAMDB and MolDdatabases for atomic and molecular collisional and radiative processes: Belgrade nodes of VAMDC. 2017, Eur. Phys. J. D, 71, 158

Mashonkina, L., Atomic data necessary for the non-LTE analysis of stellar spectra. 2009, Phys. Scr., 134, 014004

Mihajlov, A., Sakan, N., Srećković, V., \& Vitel, Y., Modeling of the continuous absorption of electromagnetic radiation in dense Hydrogen plasma. 2011, Open Astron., 20, 604

Mihajlov, A. A., Ignjatović, L. M., \& Dimitrijević, M. S., Processes of (n - n’)-mixing in collisions of Rydberg $\mathrm{H}^{*}(\mathrm{n})$ atoms with $\mathrm{H}(1 \mathrm{~s})$ in the Solar atmosphere. 2005, Astron. Astrophys., 437, 1023

Mihajlov, A. A., Ignjatovic, L. M., Djuric, Z., \& Ljepojevic, N. N., The rate coefficients for the processes of (n - n')-mixing in collisions of Rydberg atoms $\mathrm{H}^{*}(\mathrm{n})$ with $\mathrm{H}(1 \mathrm{~s})$ atoms. 2004, J. Phys. B, 37, 4493

Mihajlov, A. A., Ignjatović, L. M., Srećković, V. A., \& Dimitrijević, M. S. 2011, Astrophys. J., Suppl., 193, 2

Mihajlov, A. A., Ignjatović, L. M., Srećković, V. A., \& Djurić, Z., The influence of (n-n $\left.{ }^{\prime}\right)$-mixing processes in $\mathrm{He}^{*}(\mathrm{n})+\mathrm{He}\left(1 \mathrm{~s}^{2}\right)$ collisions on $\mathrm{He}^{*}(\mathrm{n})$ atoms populations in weakly ionized helium plasmas. 2008, J. Quant. Spectrosc. Radiat. Transf.,, 109, 853

Mihajlov, A. A., Srećković, V. A., Ignjatović, L. M., \& Dimitrijević, M. S., AtomRydberg-atom chemi-ionization processes in solar and DB white-dwarf atmospheres 
in the presence of (n - n')-mixing channels. 2016, Mon. Not. R. Astron. Soc., 458, 2215

Mihajlov, A. A., Srećković, V. A., Ignjatović, L. M., \& Klyucharev, A. N., The ChemiIonization Processes in Slow Collisions of Rydberg Atoms with Ground State Atoms: Mechanism and Applications. 2012, J. Clust. Sci., 23, 47

Negrete, C. A., Dultzin, D., Marziani, P., \& Sulentic, J. W., Broad-line Region Physical Conditions in Extreme Population A Quasars: A Method to Estimate Central Black Hole Mass at High Redshift. 2012, Astrophys. J., 757, 62

Netzer, H. 2013, The Physics and Evolution of Active Galactic Nuclei, Cambridge: Cambridge University Press

Osterbrock, D. E., \& Ferland, G. J. 2006, Astrophysics of gaseous nebulae and active galactic nuclei, 2nd. ed. by D.E. Osterbrock and G.J. Ferland. Sausalito, CA: University Science Books

Przybilla, N. \& Butler, K., Non-LTE Line Formation for Hydrogen Revisited. 2004, Astrophys. J., 609, 1181

Srećković, V., Dimitrijević, M., Ignjatović, L., Bezuglov, N., \& Klyucharev, A., The Collisional Atomic Processes of Rydberg Hydrogen and Helium Atoms: Astrophysical Relevance. 2018a, Galaxies, 6, 72

Srećković, V., Ignjatović, L., \& Dimitrijević, M., Symmetric Atom-Atom and IonAtom Processes in Stellar Atmospheres. 2018b, Atoms, 6, 1

Srećković, V. A., Dimitrijević, M. S., \& Ignjatović, L. M., Atom-Rydberg atom chemiionization/recombination processes in the hydrogen clouds in broad-line region of AGNs. 2018c, Mon. Not. R. Astron. Soc., 480, 5078

Srećković, V. A., Mihajlov, A. A., Ignjatović, L. M., \& Dimitrijević, M. S., Excitation and deexcitation processes in atom-Rydberg atom collisions in helium-rich white dwarf atmospheres. 2013, Astron. Astrophys., 552, A33

Srećković, V. A., Mihajlov, A. A., Ignjatović, L. M., \& Dimitrijević, M. S., Ion-atom radiative processes in the solar atmosphere: quiet Sun and sunspots. 2014, Adv. Space Res., 54, 1264

Vriens, L. \& Smeets, A. H. M., Cross-section and rate formulas for electron-impact ionization, excitation, deexcitation, and total depopulation of excited atoms. 1980, Phys. Rev. A, 22, 940 